THE sea moths are a small family (Pegasidae) of marine fishes found only in the Indian and western Pacific oceans, from East Africa to Hawaii. They are enigmatic little animals encased in a hard exoskeleton and with relatively small fins except for the pectorals which are close behind the head and which open fanning out in the horizontal plane. It is these large pectoral fins, wing-like at a first glance, which gave them the name of sea moths. In life these fins are 'fluttered' and the fish moves in short bursts over the sea bed-as W. E. Gosline and V. E. Brock (Handbook of Hawaiian fishes, 290; 1960) observe of captive fish "this species acts

like a not very large butterfly". Curiously for such interesting-looking animals very little seems to be known of their natural history. Most seem to live in shallow water (down to $200 \mathrm{~m}$ ) on sandy bottoms, presumably relying on cryptic coloration and irregular swimming action to avoid predators, although one species is described as being red in colour with blue eyes!

While their biology and life style are poorly known there is a wealth of discussion. and disagreement, over their systematic position. Recently, T. W. Pietsch (Copeia (3) 517 ; 1978) has reviewed the previous literature and on the basis of his studies of the skeleton of the sea moths has at last provided firm evidence for their classification. As Pietsch points out two main schools of opinion have existed in the past. The earliest discussions placed them with the pipefishes. sea horses, and their relatives. a stance that was general up to the early twentieth century. However. Günther (An Introduction to the Study of Fishes, 482-483; 1880) and

\title{
Sea moths pinned down
}

from a Correspondent

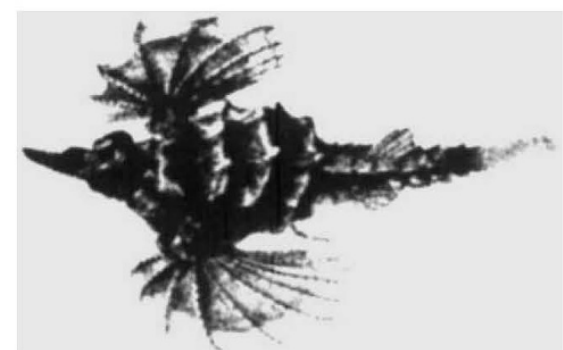

Fig. 1 Pegasus draconis. After Day, 1878.

others, impressed by resemblances to certain scorpaeniform fishes (gurnards. scorpion fishes, and especially the poachers) aligned them with this group. Later authors followed this lead, some more tentatively than others, but the general consensus of opinion during the present century has been that they were a distinct order, Pegasiformes, close to but distinct from the scorpaeniform stem.

As a result of an elegant study of the skeleton of Pegasus, Pietsch has shown that its upper jaw in particular seems to be unique amongst living fishes although similar, if not identical, to the jaw mechanism of the fossil Ramphosus from the Lower Eocene of Monte Bolca and Denmark. There has been general agreement that most of the structures of Ramphosus place it amongst the syngnathiform fishes (such as pipefishes). This suggested that Pegasus may well be closely allied to the pipefishes and their allies and Pietsch has found a number of significant features which support this view. In addition to the similarities in cranial osteology. the most anterior vertebrae are elongate which is a feature typical of the syngnathiform fishes; also the gill filaments of Pegasus are tufted and lobe-like, not laminate as in most fishes, again a feature of the pipefishes. Pietsch argues convincingly from these and other features that the true relationships of the sea moths lie in fact with the pipefishes and their allies, not with the scorpaeniform fishes.

His further conclusions regarding the classification of these enigmatic little fishes is that they lie intermediate between the two main groups, on the one hand the sticklebacks (themselves a group with a chequered history of classification) and on the other the pipefishes and their allies. All three groups are now united in the order Gasterosteiformes. To allow for the absorption of the new group within the order Pietsch has put forward a new classification in which the sea moths represent a new superfamily. As he points out, this is tentative, as further investigation of several presumed relatives is still required.

It is mildly ironic that these littleknown fishes, early examples of which mostly reached Europe as pinned specimens in Chinese insect boxes, have now been placed alongside the sticklebacks and pipefishes which are probably the most intensively studied fishes. The cynic might also point out that in this new classification the sea moths are merely returning to the position they occupied in the midnineteenth century. However, on account of Pietsch's careful anatomical comparisons and detailed discussion it seems that this time they will be firmly pinned down-not in insect boxes-but in between the sticklebacks and the pipefishes within the order Gasterosteiformes. between donor and recipient cells. Although mating signals have been postulated in conjugation little convincing proof has yet been obtained for the presence of specifically 'sexual' attractants. A recent paper from D. Clewell's laboratory (Dunny. Brown \& Clewell, Proc. natn. Acad. Sci. U.S.A. 75,$3479 ; 1978$ ) now provides evidence for the existence of a sex pheromone in the Gram-positive bacterium Streptococcus faecalis, a normal inhibitant of the gut. Some strains of this organism possess plasmids specifying a number of characteristics including haemolysin production. hacteriocin production and antibiotic resistance. Certain of these plasmids can promote gene transfer by

J. R. Saunders is a Lecturer in the Department of Microbiology. University of Liverpool. a process requiring cell-to-cell contact and resembling conjugation in Gramnegative bacteria (Jacob \& Hobbs 1974; J. Bact. 117, 360; 1974). It has been found that donor and recipient strains of $S$. faecalis form clumps in mating mixtures. Clumping has the effect of bringing donors and recipients into close contact with each other and obviously increases the probability of successful transfer of DNA during conjugation. The formation of these clumps or mating aggregates in $S$. faecalis is apparently induced by an extracellular, heat stable protein of molecular weight between 1.000 and 10.000 . This protein, termed a clumping inducing agent (CIA) is produced. perhaps surprisingly. only by recipient (female) cells and not by donors (males). Furthermore, cell-free preparations of CIA will cause donor cells to adhere even in the absence of recipients. However, CIA is not a physical agglutinating agent but rather a signal (pheromone?) which induces in donors a sequence of events resulting in cellular adhesion. Donor cells must be exposed to CIA for $30 \mathrm{~min}$ before clumps are formed. During this time the CIA seems to induce the synthesis of RNA and protein species which are necessary for clumping.

A further interesting property of the $S$. faecalis mating system is that recipients show a reduced ability to elicit CIA once they have acquired a selftransmissible plasmid thereby becoming male. This implies that streptococcal sex factors can regulate CIA either by switching off sunthesis of the protein or by inactivating pre-existing molecules of CIA. This would seem to be reasonable in terms of biological econ- 